\title{
OSTEOPATHIC MANIPULATIVE TREATMENTS FOR PEDIATRIC CONDITIONS
}

\author{
Selena G. M. Raines, MPH, DO'; Amanda L. Ramey, DOำ \\ 1 St. Claire Health Care, Morehead, KY
}

KEYWORDS:
Asthma
Osteopathic
Manipulation
Osteopathic
Manipulative Treatment
Otitis Media
Pediatric

ABSTRACT:

Osteopathic manipulative treatment (OMT) has been used in the treatment of pediatric patients for decades. The authors performed a systematic review to find evidence showing the safety and efficacy of OMT use in pediatric patients for common pediatric conditions seen in the outpatient setting. The conditions reviewed are otitis media and asthma, as they are the most common acute reason children under age five present to their physician and the most common chronic condition affecting children, respectively. ${ }^{1,2}$ The review found evidence of OMT being beneficial in the reduction of middle ear effusions following otitis media. ${ }^{3}$ The use of OMT was also shown to increase the peak expiratory flow of children with asthma. ${ }^{4}$ Very few studies have been performed to show the safety of osteopathic manipulative treatment; however one study showed that the incidence of iatrogenesis is minimal. Several easy-to-use techniques are demonstrated in the hopes that this will encourage more osteopathic physicians to incorporate the use of OMT into daily practice. As it stands, more studies are needed to give statistical significance and to prove that OMT is a safe, effective, non-invasive option for the treatment of various pediatric conditions.

\section{INTRODUCTION}

When reviewing the literature, there is a significant amount of information about the use of osteopathic manipulativetreatment (OMT) in certain sects of the pediatric population, particularly in newborns and preterm infants. There are also several resources filled with case-based and retrospective evidence for the use of OMT in the pediatric population from some of the pioneers in the field; Viola Frymann, DO and Beryle Arbuckle, DO. A couple of specific patient populations, those with cystic fibrosis and cerebral palsy, are represented in the literature concerning the use of OMT in disease treatment and management. However, the authors wanted to determine what research is available to show the safety and efficacy of using OMT as part of the treatment of common pediatric conditions in the outpatient setting and what common techniques can be used in the treatment of these patients.

\section{METHODS}

First, the authors determined the most common pediatric diagnoses made in the outpatient setting based on ICD-10 codes. A list of the 25 most used codes was narrowed to more specific diagnoses and to exclude encounters for routine health examination and vaccine administration. Several diagnoses were eliminated from the list because they were general and/ or could be included in some of the other listed diagnoses. For example, streptococcal sore throat would be included with acute pharyngitis and acute upper respiratory infection, cough, fever, abdominal pain, viral infection and viral disease were felt to be too broad to be inclusive in this search. The remaining common diagnoses from the list were otitis media, acute pharyngitis, asthma, allergic rhinitis, sinusitis, dermatitis, ADHD, bronchitis, conjunctivitis, esophageal reflux, influenza, gastroenteritis/colitis, constipation and pneumonia. ${ }^{5}$ Once this list was determined, PubMed was searched for articles pertaining to the use of osteopathic manipulation in the pediatric population for each diagnosis, respectively. Only otitis media and asthma had relevant scholarly works that met these criteria and were sufficient enough to be included in this review.

\section{CORRESPONDENCE:}

Selena G. M. Raines, MPH, DO I selena.raines@st-claire.org 


\section{OTITIS MEDIA}

Otitis media is reported to be the most common reason that children under the age of five visit the doctor other than for routine wellness examinations and vaccinations. ${ }^{1}$ In the U.S. alone, it is estimated that pediatric visits for otitis media total more than 20 million per year. ${ }^{6}$ Estimates show that by two years of age, over $90 \%$ of children in the U.S. have had at least one episode of acute otitis media. In the year 2000, the U.S. estimated the economic costs associated with acute otitis media at greater than $\$ 5$ billion, with these costs not only coming from the rendering of health care itself but also the loss of revenue and productivity in terms of employment, as parents were required to stay home and care for ill children. ${ }^{3}$ The need to find effective treatments for acute otitis media and the prevention of recurrent infections stems from the fact that recurrent infections lead to the potential sequela of hearing loss, delays in language and behavior, barriers to education, and disorders in the vestibular system and motor function. ${ }^{6}$

With otitis media being the most common acute diagnosis in the pediatric patient, it was not surprising that this search yielded the most results for clinical research. The Degenhardt and Kuchera study ${ }^{6}$ noted that the use of OMT in treating children with acute otitis media appeared to be beneficial since $62 \%$ of their study participants did not have a recurrence of acute otitis media( AOM) at one year, but the small sample size $(\mathrm{N}=8)$ was not enough to claim effectiveness. Their study was also limited by the lack of a control group and the fact that the investigators did not use a specific treatment protocol and instead treated the somatic dysfunctions identified on any given day, making the reproduction of the results impossible. Their approach to treatment was the use of balanced membranous tension or osteopathic cranial manipulative medicine and myofascial release focusing on the head and face, neck, thorax, ribcage, lumbar region and pelvis. ${ }^{6}$

In a separate study, Steele et $a^{\beta}$ conducted a prospective, randomized, blinded, controlled study using a standardized OMT protocol, in addition to standard of care, to determine the efficacy of such treatment on the resolution of the middle ear effusion following an AOM. ${ }^{3}$ Their standardized protocol consisted of nine techniques using the modalities of myofascial release, balanced ligamentous tension, and osteopathic cranial manipulation targeted at the head and neck, ribcage and diaphragm, the pelvis, and the thoracolumbar junction. The investigators gave specifics concerning patient positioning and physician hand placement, giving the better potential for reproducibility of the findings. While the number of patients enrolled in this study was still fairly low $(\mathrm{N}=43)$, they were able to show, with statistical significance $(p=0.02)$, that the use of an OMT protocol can reduce the presence of middle ear effusion following acute otitis media and can prevent the need for surgical intervention. ${ }^{3}$

In the study by Steele et $a{ }^{\beta}$, the investigators mention that each treatment protocol lasted anywhere from 15-30 minutes, which might be effective in the research setting, but it is not efficient for use in acute, outpatient visits where patients are often scheduled every 10-15 minutes. Considering that eustachian tube dysfunction, because of its more horizontal positioning in pediatric patients, plays a large role in the development of AOM, it may be more efficient to target this structure when incorporating the use of OMT in the outpatient clinic. Attention could also be given to the anterior cervical muscles and fascia and the thoracic inlet since this is the path of the lymphatics that drain the middle ear. Considering the lymphatic component in disease management, the rib cage and diaphragm would also be an easy target for treatment, as the diaphragm and thoracic cylinder act as a constant lymphatic pump. ${ }^{7}$

In 2009 the Osteopathic Cranial Academy published a position paper on the use of OMT for treatment of otitis media in which they concluded that antibiotic overprescribing and unnecessary surgical intervention are often seen in the treatment of otitis media, with little to no long-term benefit. They concluded that OMT should be the first-line treatment in the majority of cases of otitis media and should be used as part of the treatment protocol in all cases. ${ }^{8}$ Given that the other accepted treatments to prevent recurrence are prophylactic antibiotics or surgical interventions, OMT should be considered a safe and non-invasive method of preventing the long-term sequela associated with recurrent otitis media and chronic middle ear effusion.

\section{ASTHMA}

Asthma is the most common chronic condition affecting children. ${ }^{9}$ An estimated $6 .{ }^{1}$ million children have been diagnosed with asthma and annual health care costs associated with childhood asthma are estimated at $\$ 56$ billion. The prevalence of pediatric asthma ranges from $5-12 \%$ of the pediatric population in the U.S. ${ }^{2}$ Asthma is one of the main causes of hospitalization in children under the age of five and the incidence has been increasing over the last few years. ${ }^{9}$ Asthma is generally a lifelong disease increasing the risk of chronic obstructive pulmonary disease later in life.

Even though asthma is a common condition encountered in the pediatric population, there is much less research on incorporating OMT into treatment protocols. In a randomized controlled trial conducted by Guiney et $a l^{4}$, they showed a statistically significant (95\% confidence interval) increase in the peak expiratory flow of asthmatic patients treated with OMT versus sham treatment. Of note, this study was conducted on stable, established patients in an asthma clinic and did not include patients experiencing acute distress. At the onset of the study, the group hypothesized that the use of OMT could improve overall respiratory function by decreasing somatovisceral reflexes and maximizing the function of the rib cage and thorax. The techniques used in this study were listed as rib raising, myofascial release techniques and muscle energy for the rib cage with the authors citing Foundations for Osteopathic Medicine as a reference for the techniques used but giving no further details about the treatment protocol. The limitations of this study include a small sample size $(\mathrm{N}=140)$ and the fact that multiple physicians were performing the OMT, leading to possible variations in the delivery of the treatments. ${ }^{4}$ The literature also includes both a review article and clinic practice article that make a case for using OMT to treat asthma patients but neither of these gives any data or treatment specifics. ${ }^{10,11}$

The musculoskeletal system plays a key role in respiratory function. While there are many pharmacologic agents proven to 
treat asthma effectively, these medications do nothing to affect the musculoskeletal system. The use of OMT, as a safe and non-invasive treatment, could help to provide a comprehensive treatment protocol for pediatric asthma patients by addressing this musculoskeletal component.

\section{SAFETY}

Patient safety is always a concern when performing any type of procedure and OMT is no different. Parents are often apprehensive when it comes to treatments being performed on their children, so it would be helpful to be able to confidently, and with the backing of clinical evidence, reassure them that OMT is safe to use in the pediatric population. None of the clinical trials discussed thus far reported any adverse events experienced as a result of treatment, but with very small numbers in these trials, this may not prove safety.

To provide more evidence supporting the safe use of OMT in the pediatric population, Hayes and Bezilla ${ }^{12}$ conducted a retrospective review of medical records to determine the incidence of iatrogenesis when OMT was performed on this population. They reviewed records looking for evidence of aggravations and complications. Aggravation was defined as patient complaints after treatment or a worsening of symptoms. Complication was defined as dislocation, fracture, sprain or strain, cerebrovascular accident, pneumothorax or death. Their review of 346 medical records showed zero documented complications and 31, or $9 \%$, documented aggravations from treatment. Some of the common aggravations were soreness, pain, headache and irritability. Within 48 hours of the aggravation, seven of those 31 patients reported complete resolution of the aggravation symptoms. The investigators noted that some of these reported aggravations would be what osteopathic physicians know to be normal, posttreatment responses as the body adapts to structural changes. This study concluded that the incidence of iatrogenesis is minimal and the use of OMT in the pediatric population appears to be safe, with the caveat that more and larger studies need to be conducted to provide the best evidence to prove this conclusion. ${ }^{12}$

\section{TREATMENT TECHNIQUES}

The following techniques can be incorporated into office or acute settings to help treat patients with acute otitis media or asthma and are the authors' variations on the material presented in Somatic Dysfunction in Osteopathic Family Medicine, 2nd Edition, unless otherwise referenced. ${ }^{13}$ The techniques are not timeintensive and can be performed by osteopathic physicians who do not have an in-depth knowledge of osteopathic principles and practices as do those physicians who specialize in neuromuscular manipulative medicine. As with any case, if the patient fails to improve with these quick, targeted treatments, the authors would recommend a full osteopathic structural exam to identify somatic dysfunction, because it is not uncommon for the key lesion to be found elsewhere in the body.

\section{Auricular Drainage}

This is a lymphatic technique used to promote drainage of the middle ear by improving eustachian tube function. The physician separates the third and fourth digits on the treating hand and places these around the ear. Apply slight pressure to stabilize the ear, and then begin to move the tissues in a clockwise direction, in a rhythmic fashion. Once at the six o'clock position, emphasize inferior and medial pressure to enhance the technique, before continuing the clockwise motion. This is a technique that could easily be taught to the patient or parent to continue at home.

\section{FIGURE 1:}

Auricular drainage

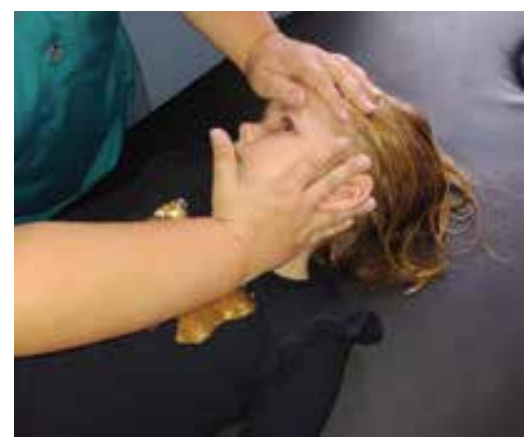

\section{Galbreath Technique}

This is another lymphatic technique used to aide in the decongestion of the middle ear and eustachian tube. The physician positions the treating hand over the angle of the mandible and uses the other hand to stabilize the head. Pressure is placed from the inferior aspect of the angle of the mandible to move this structure both medially and inferiorly effectively. Care must be taken not to put too much pressure or strain on the jaw itself to prevent inducing a dysfunction at the temporomandibular joint. This technique can also be taught to the patient or parent to continue at home. ${ }^{14}$

\section{FIGURE 2:}

Galbreath technique, supine

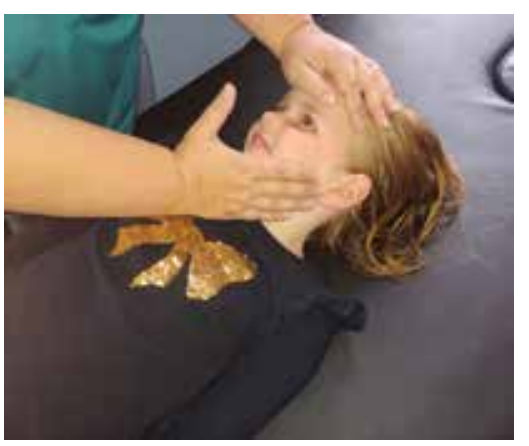




\section{FIGURE 3:}

Galbreath technique, seated

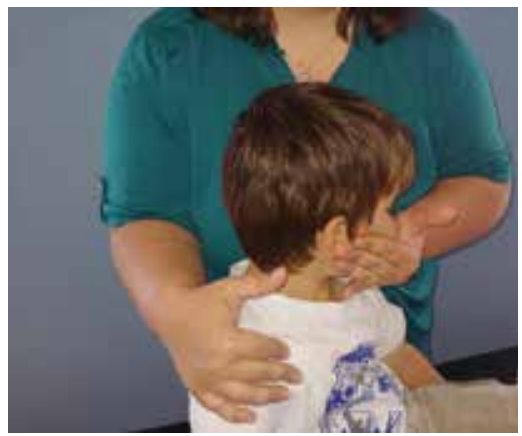

\section{Thoracic Inlet Release}

The thoracic inlet is a bony ring that is comprised of the first thoracic vertebra, the first ribs and the manubrium. The clavicle is also an important boney structure as it contacts the sternum and is a landmark for locating the first ribs anteriorly. The purpose of this technique is to loosen the fascial and soft tissue layers associated with the thoracic inlet, as they not only aide lymphatic drainage, but respiration as well. The physician uses both hands to contact the bony structures of the thoracic inlet and applies slight pressure. The physician then tests ease of movement of the fascial and soft tissues associated with the thoracic inlet in all planes of motion: flexion and extension, side-bending and rotation. The physician can choose to treat those tissues in either a direct or indirect manner. To treat the dysfunctions directly, the physician would induce motion in the directions that increase tension in the fascial and soft tissue structures. The opposite manner, inducing motion to decrease tension in the structures, would be an indirect treatment.

\section{FIGURE 4:}

Thoracic inlet release, supine

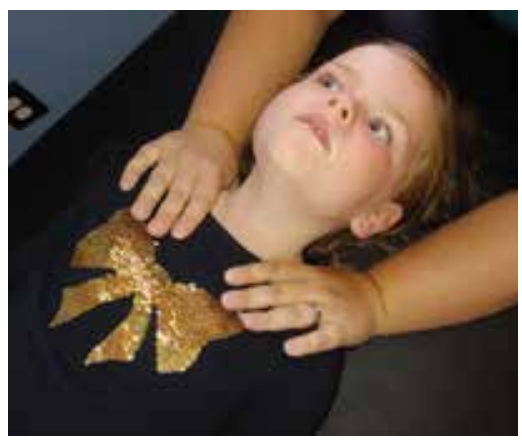

FIGURE 5:

Thoracic inlet release, seated

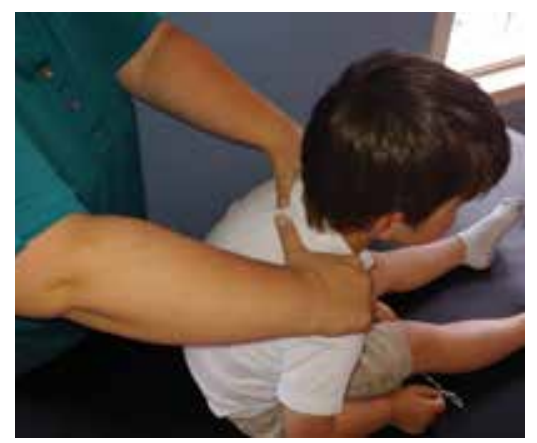

\section{Respiratory Diaphragm Release}

This technique can be used to ease any tension in the respiratory diaphragm and surrounding tissues and it will likely also affect the ligamentous structures at the costovertebral junctions of the lower rib cage. The physician places both hands on the lower rib cage, wrapping around to the axillary line. The thumbs are midline, at the distal end of the sternum, taking care not to put pressure on the xiphoid process. The physician tests ease of movement of the diaphragm muscle and surrounding tissues in all planes of motion: flexion and extension, side-bending and rotation. The physician then induces motion in those three planes for either a direct or indirect treatment and waits for the tissues to ease. The authors find that often a slight compressive force between the two hands will aide in this process.

\section{FIGURE 6:}

Respiratory diaphragm release, supine

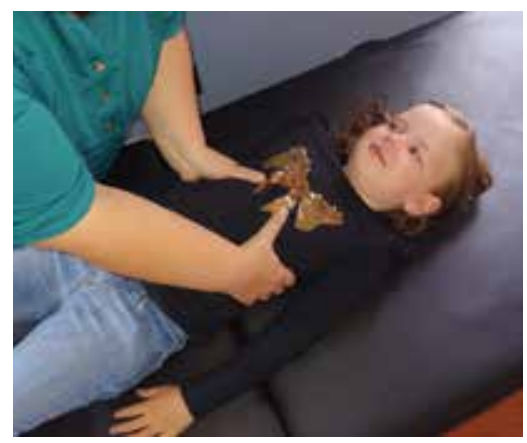

FIGURE 7:

Respiratory diaphragm release, supine

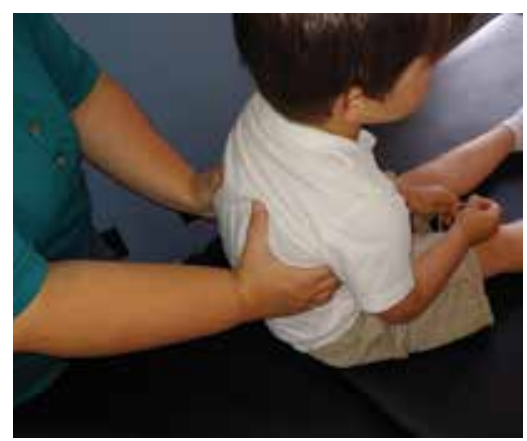




\section{Rib Raising}

This technique not only induces motion into the costovertebral joints, but it also adds stimulus to the sympathetic chain ganglia located along the thoracic spine. The physician places the finger pads of both hands along with the thoracic paraspinal muscles and posterior rib angles. The physician then applies an anterior and lateral pressure, using their forearms as a long lever. This is held until tissue ease and is repeated until the entire length of the costovertebral junctions is treated bilaterally.

\section{FIGURE 8:}

Hand placement for rib raising

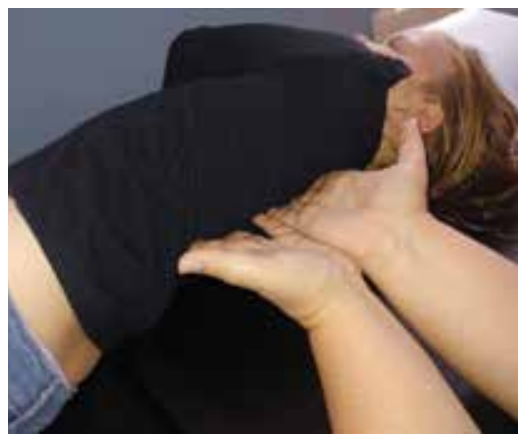

FIGURE 9:

Rib raising, supine

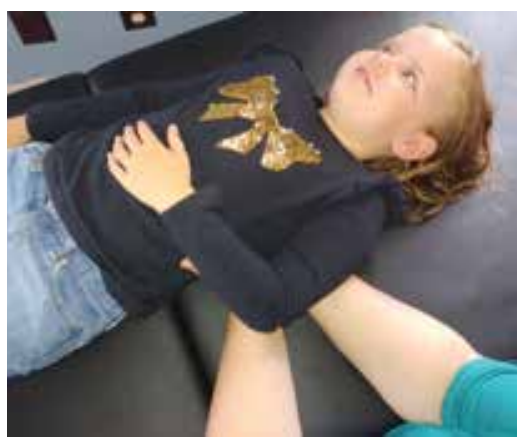

Any of these techniques can be performed with the patient seated or supine, depending on patient comfort and cooperation. The authors find that many toddler-aged children are less cooperative and often want to remain in the arms of a parent. With some creative positioning all of these techniques can be performed while a parent holds the child.

\section{CONCLUSION}

OMT could prove to be a safe, effective and non-invasive treatment option for many of the common problems seen in outpatient pediatrics. The small studies reviewed here show patient benefits when OMT is incorporated into treatment and the osteopathic community has numerous examples of case reports and anecdotal evidence showing benefit, but larger studies are needed to prove efficacy. This review also shows many areas of research opportunity for the osteopathic community, as the majority of the most common diagnoses encountered in pediatric outpatient visits could not be found anywhere in the literature concerning studies showing the use of OMT in treatment and management of these patients. Osteopathic manipulative treatment is considered generally safe for use in the pediatric patient, but again, more studies are needed to give statistical proof to this statement. The osteopathic community must work diligently to produce quality research to prove that the use of OMT is a safe and effective adjunctive treatment for pediatric patients with a variety of presenting complaints and conditions.

\section{AUTHOR DISCLOSURES:}

No relevant financial affiliations or conflicts of interest. If the authors used any personal details or images of patients or research subjects, written permission or consent from the patient has been obtained.

\section{RESOURCES:}

1. Winger K, Hendriksz T, Wolf K, Talsma J, Pierce-Talsma S. Osteopathic Manipulative Treatment for Pediatric Patients With Otitis Media. J Am Osteopath Assoc. 2020; 120 (3): e5-e6.

2. American Lung Association. Asthma and Children Fact Sheet. https:// www.lung.org/lung-health-diseases/lung-disease-lookup/asthma/learnabout-asthma/asthma-children-facts-sheet. Published February 27, 2020. Accessed March 16, 2020.

3. Steele KM, Carreiro JE, Viola JH, Conte JA, Ridpath LC. Effect of Osteopathic Manipulative Treatment on Middle Ear Effusion Following Acute Otitis Media in Young Children: A Pilot Study. J Am Osteopath Assoc. 2014; 114(6): 436-447. Doi:10.7556/jaoa.2014.094

4. Guiney PA, Chou R, Vianna A, Lovenhiem J. Effects of Osteopathic Manipulative Treatment on Pediatric Patients With Asthma: A Randomized Controlled Trial. J Am Osteopath Assoc. 2005; 105(1): 7-12.

5. A New Top 25? AAP Pediatric Coding Newsletter. 2013; 8(11): 4-5.

6. Degenhardt BF, Kuchera ML. Osteopathic Evaluation and Manipulative Treatment in Reducing the Morbidity of Otitis Media: A Pilot Study. J Am Osteopath Assoc. 2006; 106:327-334.

7. Carreiro, Jane. Pediatric Manual Medicine: An Osteopathic Approach. Edinburgh: Churchill Livingstone/Elsevier; 2009: 80-81.

8. Dolgin E. Otitis Media- The Cranial Academy Position Paper. Osteopathic Cranial Academy; 2009. http://www.osteohome.com/resources/Articles/ OtitisMediaPP.pdf. Published November 2009. Accessed March 12, 2020.

9. Ferrante G, La Grutta S. The Burden of Pediatric Asthma. Frontier Pediatrics. 2018; 6:186

10. Paul FA, Buser BR. Osteopathic manipulative treatment applications for the emergency department patient. J Am Osteopath Assoc. 1996; 96(7): 403-409.

11. Rowane WA, Rowane MP. An osteopathic approach to asthma. J Am Osteopath Assoc. 1999; 99(5): 259-264.

12. Hayes NM, Bezilla TA. Incidence of Iatrogenesis Associated With Osteopathic Manipulative Treatment of Pediatric Patients. J Am Osteopath Assoc. 2006; 106: 605-608.

13. Nelson, Kenneth. Somatic Dysfunction in Osteopathic Family Medicine. 2nd ed. Philadelphia, PA: Wolters Kluwer Health; 2015.

14. Pratt-Harrington, Dale. Galbreath technique: a manipulative treatment for otitis media revisited. J Am Osteopath Assoc. 2000; 100(10): 635-639. 